\title{
Casuística de perezosos (Bradypus variegatus y Choloepus hoffmanni) recibidos en la Unidad de Rescate y Rehabilitación de Animales Silvestres (URRAS) de la Universidad Nacional de Colombia entre 2005 y 2010
}

\author{
Daniel Mauricio Duarte Riaño ${ }^{\mathrm{A}}$, Natalia Pradilla Villamizar ${ }^{\mathrm{A}}$ Y \\ Claudia Isabel Brieva Rico ${ }^{\mathrm{A}, 1}$
}

\begin{abstract}
A Facultad de Medicina Veterinaria y de Zootecnia, Universidad Nacional de Colombia, Carrera 30 \# 45-03, Edificio 481, Unidad de Rescate y Rehabilitación de Animales Silvestres (URRAS), Bogotá, Colombia. E-mail: damduarteri@unal.edu.co (DMDR), npradillav@unal.edu.co (NPV), cibrievar@unal.edu.co (CIBR)
\end{abstract}

${ }^{1}$ Autor para correspondencia

\begin{abstract}
Resumen Doce perezosos de tres o dos dedos (Bradypus variegatus y Choloepus hoffmanni, respectivamente) ingresaron a la Unidad de Rescate y Rehabilitación de Animales Silvestres (URRAS) de la Universidad Nacional de Colombia para su diagnóstico y rehabilitación. Se extrajeron datos de su historia clínica completa, como el motivo de consulta, características del cautiverio, patologías presentadas y desenlace del caso, entre otros. Se encontró que la mayoría de enfermedades correspondían a patologías del sistema tegumentario, respiratorio o digestivo, asociadas principalmente a las condiciones inadecuadas de cautiverio en las que se encontraban los animales antes de su ingreso.
\end{abstract}

Palabras clave: cautiverio, Folivora, patologías, Pilosa, rescate, Xenarthra

Retrospective study of sloths (Bradypus variegatus and Choloepus hoffmanni) treated at the Wildlife Rescue and Rehabilitation Unit (URRAS) of the Universidad Nacional de Colombia between 2005 and 2010

\begin{abstract}
Twelve three-toed and two-toed sloths (Bradypus variegatus and Choloepus hoffmanni, respectively) were admitted to the Wildlife Rescue and Rehabilitation Unit (URRAS) of the National University of Colombia between the years of 2005 and 2010 for diagnosis and rehabilitation. Data were extracted from complete clinical records. Information included, among others, cause of admission, characteristics of the enclosure in captivity, vital signs, observed pathologies, and case resolution. The majority of diseases were pathologies of the integumentary, respiratory or digestive system. They were mainly associated to poor husbandry while in captivity before the animal's admission to URRAS.
\end{abstract}

Keywords: captivity, Folivora, pathologies, Pilosa, rescue, Xenarthra

\section{INTRODUCCIÓN}

Los perezosos son animales pertenecientes al superorden Xenarthra, orden Pilosa y suborden Folivora (Gardner, 2008). En Colombia se encuentran representados principalmente por dos especies: Bradypus variegatus (perezoso de tres dedos) y Choloepus hoffmanni (perezoso de dos dedos). Estos animales son presa fácil del comercio ilegal de fauna silvestre debido a su aparente temperamento dócil. Los traficantes suelen limar sus uñas y dientes e incluso mantenerlos sedados para hacerlos más atractivos a los ojos del comprador (Plese, 2014). No es extraño encontrarlos fuera de su hábitat natural, ya sea comercializados como mascotas, ubicados en parques para entretener a los visitantes, vendidos en mercados públicos como alimento o sacrificados para la venta de sus partes en la fabricación de medicinas (Plese et al., 2008). Sin embargo, la principal amenaza para estos animales es la deforestación, la cual causa la degradación y fragmentación severa de sus hábitats (Ballesteros et al., 2009).

Muchas de las patologías que afectan a estas especies se deben a la manipulación inadecuada que sufren durante su captura, transporte y cautiverio, 
$\mathrm{y}$ en algunos casos al abandono al que son sometidos. Es común que presenten problemas como traumatismos y desórdenes digestivos, producto de las condiciones ambientales inadecuadas en las que son mantenidos (Messias et al., 2001; Plese et al., 2008). Por estos motivos, los perezosos ingresan de manera relativamente frecuente a los centros de rescate de fauna silvestre como la Unidad de Rescate y Rehabilitación de Animales Silvestres de la Universidad Nacional de Colombia (URRAS), localizada en la ciudad de Bogotá, Colombia.

En este estudio retrospectivo se señalan y analizan las causas más comunes de consulta, los hallazgos al examen clínico, las patologías presentadas con mayor frecuencia y el destino final de los perezosos atendidos en URRAS entre 2005 y 2010.

\section{MATERIALES Y MÉTODOS}

Se realizó una búsqueda en los archivos consecutivos de URRAS de las historias clínicas de perezosos de dos y tres dedos que ingresaron entre los años 2005 y 2010. En total se obtuvieron 12 historias clínicas. De cada una se extrajo información sobre la especie, Estado de Desarrollo Biológico (EDB), sexo, procedencia, tiempo de cautiverio, características del cautiverio, dieta suministrada, motivo de consulta, signos clínicos y duración, frecuencia cardíaca en pulsaciones por minuto (ppm), frecuencia respiratoria en respiraciones por minuto (rpm), temperatura rectal, temperamento, actitud, condición corporal, color de las membranas mucosas, tiempo de llenado capilar, peso, patologías presentadas y desenlace del caso. El término EDB se utiliza ampliamente en Colombia para referirse al rango de edad al que pertenece el animal (infantil, juvenil, subadulto, adulto, geriátrico; Varela, 2009). Al ser un estudio retrospectivo no se tuvo en cuenta la metodología empleada por los clínicos al realizar las valoraciones iniciales, ni los criterios de determinación del sexo o la edad al momento de la admisión; no obstante, se aclara que URRAS cuenta con un formato de historia clínica estandarizado que sigue el método del problema orientado, utilizado en la enseñanza veterinaria en la Facultad de Medicina Veterinaria y de Zootecnia de la Universidad Nacional de Colombia. Por lo tanto, se tiene certeza de que se utilizó la misma metodología para todos los individuos aquí analizados.

Se organizó y analizó la información recabada según especie, grupo etario y sexo. Los datos de mantenimiento en cautiverio se recolectaron y se correlacionaron con el estado de salud de los especímenes. Se organizaron datos clínicos para cada animal, se listaron las patologías presentadas y se clasificaron de acuerdo a su frecuencia de presentación. Finalmente se analizó el destino final de los animales (sobrevivencia o fallecimiento).
Los datos obtenidos se analizaron con estadística descriptiva, utilizando el programa Microsoft Excel (Microsoft Corporation, Redmond, Estados Unidos).

\section{RESULTADOS}

La mayoría de los perezosos rescatados, 83,3\% (10/12), eran infantiles o juveniles (TABLA 1). De la especie $B$. variegatus se recibieron dos individuos infantiles y tres juveniles, y de C. hoffmanni dos infantes, tres juveniles y dos adultos. Sólo en el 25\% (3/12) de los animales se logró establecer el sexo, registrándose una hembra y un macho de $B$. variegatus y tres animales de sexo indeterminado. Para C. hoffmanni se registró una hembra y seis animales de sexo indeterminado.

Respecto a los lugares de origen, se encontró que los animales fueron trasladados desde diferentes departamentos del país: Cundinamarca (cuatro C. hoffmanni y dos B. variegatus), Boyacá (un C. hoffmanni), Bolívar (dos B. variegatus), Antioquia (un B. variegatus) y Tolima (dos C. hoffmanni).

Con relación al tipo de cautiverio en el que se encontraban los perezosos antes de ingresar a URRAS, se reportaron diferentes condiciones de mantenimiento: cajas y cobijas, "mamá canguro" (mantener al animal en el pecho de una persona todo el tiempo), libertad en casa, ubicados sobre las plantas ornamentales dentro del hogar, y dentro de cajas de transporte tipo Kennel.

Los alimentos que se ofrecieron a $\operatorname{los} B$. variegatus durante su cautiverio ilegal incluyeron leche deslactosada y descremada, suero lácteo, suero oral y hojas de brevo (Ficus carica). A los individuos de $C$. hoffmanni les fueron ofrecidos en su cautiverio ilegal frutas varias (mango, manzana, guayaba, banano, papaya, uva y durazno) hortalizas (tomate, repollo y lechuga), hojas de roble y suero oral de la marca Pedialyte (Abbott Laboratories de Colombia, Bogotá, Colombia). Resalta un caso en el que se suministró al animal comida casera (empanada, snacks comerciales para consumo humano de maíz inflado y queso, bebida gaseosa, papas, arroz y jugo de fruta).

Los principales motivos de consulta por los que los animales ingresaron inicialmente a URRAS fueron depresión y anorexia. En el examen clínico la mayoría de los animales estaban alertas (TABLA 1). La temperatura corporal de infantiles y juveniles de ambas especies fue similar. En general los C. hoffmanni tenían una frecuencia cardíaca más elevada que B. variegatus; esta tendencia se observó en todos los grupos etarios (TABLA 1). En ambas especies, la frecuencia respiratoria fue levemente más baja en infantes que en juveniles pero los valores fueron similares en perezosos de dos y tres dedos (TABLA 1).

En la mitad de los ejemplares (6/12) se diagnosticaron afectaciones del sistema tegumentario; el 


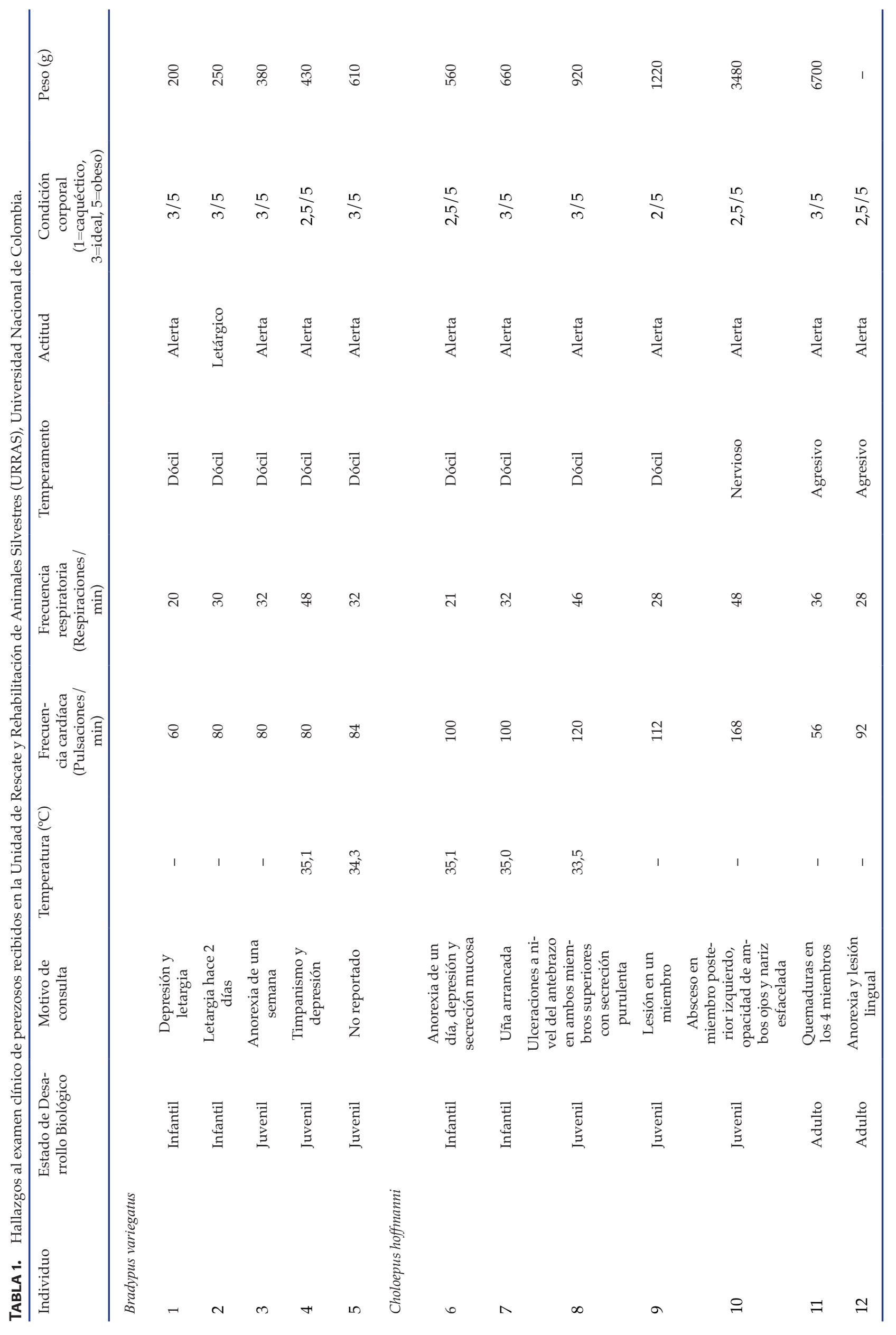


mismo porcentaje correspondió a problemas respiratorios. Las patologías digestivas afectaron al 41,6\% de los animales (5/12), las musculoesqueléticas al 16,6\% $(2 / 12)$ al igual que las oculares, y el mismo número de animales examinados (2/12) no presentó hallazgos anormales al examen clínico.

Dentro de las patologías tegumentarias se encontraron animales con úlceras cutáneas (2), descamación palmar (1), quemaduras (1), erosiones faciales (1), dermatitis de origen desconocido (1), absceso subdérmico (1) y desprendimiento de uñas (1). Los animales con patología respiratoria presentaron neumonía/ infección del tracto respiratorio bajo (5) y rinitis (1). Las patologías observadas en animales con cuadros digestivos fueron timpanismo (3), impactación (1), obstrucción gástrica (1) y laceración lingual y palatina (1). Dos animales sufrieron patologías musculoesqueléticas; un animal con fractura de mandíbula falleció a pesar del tratamiento quirúrgico realizado. Otro animal sufrió un accidente al enredar su miembro anterior con una cuerda, lo que generó isquemia y deficiente drenaje linfático del miembro; esta lesión se resolvió satisfactoriamente luego de un tratamiento antiinflamatorio. Entre las patologías oculares se encontraron opacidad corneal (1) y úlcera corneal (1). El único individuo que presentaba una patología linfática sufría de isquemia y obstrucción de drenaje linfático por compresión.

El porcentaje de animales sobrevivientes fue de $58,3 \%$ (7/12), de los cuales el 57,2\% (4/7) eran C. hoffmanni y el 42,8\% (3/7) B. variegatus. De los animales fallecidos (5/12), el 60\% (3/5) era C. hoffmanni y el $40 \%$ (2/5) B. variegatus. A los animales fallecidos se les practicó necropsia en tres de cinco casos; en los dos restantes se consideró que la causa de muerte ya estaba esclarecida con los hallazgos clínicos. De los siete animales sobrevivientes, cinco fueron trasladados al único centro de rescate especializado en xenartros en Colombia (Fundación AIUNAU) para continuar con su proceso de rehabilitación y posterior liberación. Dos fueron liberados directamente en cercanías a Bogotá porque esa fue la procedencia comprobada.

\section{Discusión}

El hecho de que la mayoría de animales recibidos pertenecieran a las categorías infantil y juvenil puede deberse a que en este estado de desarrollo los perezosos son más vulnerables y de mayor atractivo para el comprador debido a su docilidad y aspecto encantador, lo que los hace presas más fáciles y apetecidas del tráfico ilegal (Plese et al., 2008).

El lugar de procedencia de los perezosos no necesariamente coincidió con el lugar de captura; en algunos casos se relaciona con el lugar donde el animal fue encontrado o adquirido. Bradypus variegatus se encuentra distribuido naturalmente en regiones costeras de Colombia, algunas localidades bajas de los valles interandinos y en las regiones del Orinoco y Amazonas (Plese et al., 2008; Ballesteros et al., 2009). Algunos de los animales de esta especie que ingresaron a URRAS provenían de departamentos localizados en regiones apartadas de Bogotá, lo que refleja el desplazamiento que sufrieron desde sus hábitats naturales hasta regiones más centrales del país. La distribución geográfica de C. hoffmanni incluye el centro del país (Plese et al., 2016), lo que explicaría por qué ingresaron más individuos de esta especie que de $B$. variegatus.

En sólo unos pocos animales se logró determinar el sexo, lo que puede explicarse por su edad y la falta de experiencia de las personas que los recibieron. El reconocimiento de los genitales externos puede ser difícil debido a que los perezosos poseen una estructura denominada "pseudo-cloaca" por algunos autores (Taube et al., 2001). Además, las especies presentes en Colombia, a diferencia de otras como B. tridactylus, no muestran dimorfismo sexual evidente (Hayssen, 2009). Se puede recurrir a la laparotomía o técnicas de laboratorio más avanzadas como la determinación por PCR (Murata \& Masuda, 1996), aunque estas alternativas son invasivas o muy costosas. Por ello se recomienda el entrenamiento en el reconocimiento de los órganos genitales.

Es difícil analizar si el peso y la condición corporal de los animales al momento de su ingreso eran los ideales, ya que los estudios de estos rangos en las dos especies son muy escasos. En este trabajo se encontró que los animales presentaban un peso normal, teniendo en cuenta que algunos autores indican que los animales del género Choloepus son más pesados que los del género Bradypus (Emmons \& Feer, 1997).

La frecuencia cardíaca de los ejemplares de $B$. variegatus estaba dentro del rango reportado por Gilmore et al. (2000; 60-110 ppm) y levemente superior al rango registrado por DiDio (1968; 45-71 ppm). Casi todos los perezosos de tres dedos tenían una frecuencia respiratoria elevada en comparación con los valores de referencia (5-21 rpm; Gilmore et al., 2000). Sólo el juvenil de C. hoffmanni que presentaba señales de nerviosismo tenía la frecuencia cardíaca elevada comparado con los rangos normales reportados por Gilmore et al. (2000; 70-130 ppm). La frecuencia respiratoria estuvo dentro del rango publicado (10-18 rpm; Gilmore et al., 2000). En ambas especies, los valores elevados pueden haberse debido al estrés de la manipulación (Gilmore et al., 2000).

En algunos animales no fue posible determinar la temperatura rectal. Sin embargo, en los animales en los que se logró tomar, se encontró una temperatura dentro de los rangos normales reportados $\left(28-35^{\circ} \mathrm{C}\right.$; Aguilar \& Superina, 2015). Cabe aclarar que los xenartros presentan temperaturas corporales más bajas que otros mamíferos debido a su bajo metabolismo (Gilmore et al., 2000). Adicionalmente la variación 
normal en la temperatura corporal de los perezosos dificulta la determinación de un posible estado febril.

Las condiciones de cautiverio en que los animales fueron mantenidos antes de su ingreso a URRAS pudieron haber influido en la presentación de patologías. Diniz \& Oliveira (1999) mencionan que la incidencia de las diferentes enfermedades varía con el clima (periodo seco o de lluvias), el tiempo en cautiverio y el tipo de encierro. Reportan que el 96,4\% de las enfermedades se presentan en los primeros seis meses de cautiverio, lo que puede explicar la presentación de varias alteraciones de la salud de los perezosos en este estudio.

Los sistemas más frecuentemente afectados fueron el tegumentario y el respiratorio. Este resultado contrasta con lo reportado por Diniz \& Oliveira (1999) en un estudio retrospectivo de 34 Bradypus sp. y 17 Choloepus sp. del zoológico de São Paulo, Brasil, en el cual las patologías con prevalencias más altas eran de carácter nutricional, seguido por problemas digestivos y respiratorio. Sin embargo, es necesario aclarar que Diniz \& Oliveira (1999) no mencionaron patologías tegumentarias específicas sino que listaron algunas dentro de las afecciones de origen traumático.

Los problemas de piel son comunes debido a traumatismos durante la captura ilegal, el transporte, el abandono o el cautiverio por parte de los tenedores ilegales (Messias et al., 2001; Plese et al., 2008; Aguilar \& Superina, 2015). Las dietas inadecuadas causan malnutrición, la cual se ve reflejada en la mala condición del tegumento ya que un aporte no adecuado de ciertos nutrientes, como los oligoelementos, las vitaminas y los aminoácidos, puede afectar la salud de la piel y su función inmunológica (Mueller \& Dethioux, 2010).

En un estudio realizado en el Jardín Zoobotánico del Museo Paraense Emilio Goeldi en Belém, Brasil, se determinó que la causa de muerte del 95\% de los perezosos confiscados de comercio ilegal se relacionaba con problemas respiratorios (Messias et al., 2001). Efectivamente, las patologías de origen respiratorio fueron muy frecuentes en los animales aquí analizados. Pudieron deberse a un inadecuado manejo medioambiental o nutricional que causó un mal funcionamiento del sistema inmunitario, desencadenando la aparición de enfermedades respiratorias. La temperatura promedio de la ciudad de Bogotá y sus alrededores $\left(14,6{ }^{\circ} \mathrm{C}\right.$; IDEAM, 2014) se consideraría muy baja para especies que habitan climas medios y cálidos, como B. variegatus. Esta especie requiere una temperatura ambiente entre los 24 y $32{ }^{\circ} \mathrm{C}$ y una humedad superior al $60 \%$ para evitar la presentación de patologías respiratorias; además es muy susceptible a los cambios ambientales (Messias et al., 2001). Sin embargo, en el caso de C. hoffmanni la temperatura es similar a la de algunas zonas de clima frío en cercanías a Bogotá en las que se distribuye la especie naturalmente (Morales-Jiménez et al., 2004; Plese et al., 2008; Ballesteros et al., 2009). En consecuencia, es posible que su mantenimiento en ciudades de clima frío como Bogotá no sería tan perjudicial como en perezosos de tres dedos, al menos en el caso de ejemplares provenientes de tierras altas que están adaptados a temperaturas bajas.

La proporción de animales con patologías digestivas en el presente estudio coincidió con la reportada por Diniz \& Oliveira (1999). Como factor predisponente puede mencionarse el estrés generado por el cautiverio, la incorrecta manipulación realizada por traficantes y tenedores ilegales y las dietas inadecuadas suministradas. En condiciones normales, los perezosos defecan cada tres a cinco días, pero en condiciones de cautiverio pueden pasar hasta 10 días sin realizar ninguna deposición lo que, junto con procesos de deshidratación, puede generar constipación (Messias et al., 2001).

El segundo factor asociado con problemas gastrointestinales es la dieta. La mayoría de dietas suministradas por los tenedores ilegales fueron inadecuadas, pues contenían altos niveles de productos lácteos (leche, suero) y bajos niveles de fibra, lo que pudo finalmente desencadenar la aparición de patologías digestivas en algunos de los animales recibidos (Aguilar \& Superina, 2015). Los perezosos de tres dedos son animales con una dieta muy especializada que evolucionaron para hacer frente a un rango reducido de compuestos secundarios de plantas, principalmente hojas (Chiarello, 1998). En vida libre se alimentan de hojas de yarumo (Cecropia spp.), barrigón (Pseudobombax septenatum), roble (Tabebuia rosea), jobo (Spondias spp.), guácimo (Guazuma ulmifolia) y pequeñas cantidades de frutos de algunos de estos árboles (Mackenzie et al., 2005). Los animales del género Choloepus tienen una dieta más variada. Se alimentan de hojas de yarumo, lianas, espavé (Anacardium excelsum), poro-poro (Cochlospermum vitifolium), almendro (Prunus dulcis), mango (Mangifera indica) y jobo; flores y rebrotes de barrigón, maracuyá (Passiflora spp.) y árboles de la subfamilia Bombacoideae; y frutos de almendro, mango, membrillo (Gustavia superba) y jobo (Mackenzie et al., 2005).

En cautiverio, los perezosos de dos dedos pueden comer frutas, vegetales, alimento concentrado en pellets, huevos cocidos y queso; se recomienda suplementar su dieta con hojas, flores y frutos silvestres (Messias et al., 2001). Las dietas comerciales (concentrados) para perros pueden ser suministradas teniendo cuidado de no exceder la cantidad, ya que podrían ocasionar daño renal (Messias et al., 2001). El suministro de productos lácteos (leche deslactosada de vaca) podría ser adecuado en el caso de individuos infantiles. No obstante, la leche de vaca contiene demasiada grasa, calorías, colesterol y azúcares en comparación con la leche de cabra, recomendada por algunos autores para alimentar a los infantes de ambas especies 
de perezosos (Hadjipanayiotou, 1995; Gage, 2002). Se destaca el caso de un animal alimentado con comida casera, bebidas gaseosas y snacks altamente procesados, dieta que puede ser perjudicial para la salud de cualquier animal silvestre ya que contiene exceso de carbohidratos, conservantes y azúcares. Los productos lácteos, verduras altamente fermentables (repollo) o snacks comerciales también pudieron generar una alteración en la microbiota gástrica y, por ende, en el proceso de fermentación, lo cual llevó finalmente a la presentación de distensión abdominal en algunos de ellos (Aguilar \& Superina, 2015).

De los dos ejemplares con afectaciones musculoesqueléticos, uno falleció a pesar del tratamiento recibido mientras que el otro se recuperó. Las afecciones traumáticas son frecuentes en este tipo de animales por el manejo inadecuado durante el tráfico ilegal o por accidentes en su medio natural, algunos de ellos provocados por la tala de árboles (Messias et al., 2001). Estos autores recomiendan tratar las fracturas mediante técnicas convencionales de reducción, pero mencionan que el pronóstico por lo general es pobre. Para evitar accidentes en cautiverio, se recomienda no utilizar cuerdas ni telas que desprendan fibras como parte del enriquecimiento ambiental.

Algunos de los animales presentaron enfermedad ocular (conjuntivitis) que puede estar asociada a traumatismos o a deficiencias del sistema inmunitario por estrés. La conjuntivitis no se reporta como un problema de salud frecuente en perezosos.

La mortalidad o supervivencia de los animales dependió de las condiciones en las que llegaron a URRAS. Una mayor proporción de animales sobrevivió debido a la atención médica prestada oportunamente, pero algunos de ellos presentaron enfermedades de difícil tratamiento y resolución, como es el caso del paciente con fractura mandibular. Otros murieron luego de un deterioro súbito, posiblemente asociado al cautiverio y sus consecuencias, aunado a la poca información disponible respecto al manejo clínico y farmacológico de estas especies que en ocasiones no permite brindar la atención veterinaria ideal. Diniz \& Oliveira (1999) concluyen que el manejo adecuado y la identificación temprana de las condiciones patológicas son importantes para mantener la salud de los animales en cautiverio, lo que también se evidencia en el presente estudio.

\section{CONCLUSIONES}

Los infantes y juveniles de C. hoffmanni predominaron entre los perezosos recibidos por URRAS. Esto puede deberse a que son más afectados por el tráfico y tenencia ilegal en la ciudad de Bogotá. Sin embargo, no se puede excluir que esté relacionado con una mayor mortalidad de $B$. variegatus antes de que puedan ser rescatados.
Todos los perezosos que ingresaron a URRAS fueron víctimas de tráfico y/o cautiverio ilegal. La manipulación y el manejo por traficantes y tenedores ilegales resultó en patologías típicas de condiciones inadecuadas de cautiverio.

Es importante ampliar la investigación sobre el manejo médico de los perezosos, ya que la información disponible es escasa, lo que imposibilita en algunos casos ofrecer una atención veterinaria eficaz.

Se debe informar y educar al público en general sobre la inconveniencia de comprar y tener perezosos como mascotas, ya que no sólo se afecta gravemente el equilibrio ambiental, sino que se afecta la salud y bienestar de estos animales, disminuyendo así sus posibilidades de supervivencia durante el manejo post-decomiso.

\section{REFERENCIAS}

Aguilar, R. \& M. Superina. 2015. Xenarthra. Pp. 355-368 in: Fowler's zoo and wild animal medicine, Volume 8 (R. E. Miller \& M. Fowler, eds.). Elsevier Saunders, St. Louis. https: / / doi. org/10.1016/B978-1-4557-7397-8.00039-6

Ballesteros, J., K. Reyes \& J. Racero. 2009. Estructura poblacional y etología de Bradypus variegatus en fragmento de bosque seco tropical, CórdobaColombia. Revista MVZ Córdoba 14: 1812-1819.

Chiarello, A. 1998. Diet of the Atlantic forest maned sloth Bradypus torquatus (Xenarthra: Bradypodidae). Journal of Zoology 246: 11-19. https: / / doi.org/10.1111/j.1469-7998.1998. tb00127.x

DiDio, L. J. 1968. Myocardial ultrastructure and electrocardiograms of the sloth (Bradypus tridactylus) under normal and experimental conditions. Journal of Morphology 124: 83-103. https: / / doi. org / 10.1002/jmor.1051240106

Diniz, L. S. \& P. M. Oliveira. 1999. Clinical problems of sloths (Bradypus sp. and Choloepus sp.) in captivity. Journal of Zoo and Wildlife Medicine 30: 76-80.

Emmons, L. \& F. Feer. 1997. Neotropical rainforest mammals: A field guide. University of Chicago Press, Chicago. 396 pp.

Gage, L. 2002. Hand-rearing wild and domestic animals. Iowa State University Press, Iowa. 279 pp. https: / / doi.org/10.1002 / 9780470385005

Gardner, A. L. 2008. Mammals of South America. Volume 1: Marsupials, xenarthrans, shrews, and bats. University of Chicago Press, Chicago. 669 pp. https://doi.org/10.7208/ chicago/9780226282428.001.0001

Gilmore, D. P., C. P. Da-Costa \& D. P. Duarte. 2000. An update on the physiology of two- and 
three-toed sloths. Brazilian Journal of Medical and Biological Research 33: 129-146. https:// doi.org/10.1590/S0100-879X2000000200001

Hadjipanayiotou, M. 1995. Composition of ewe, goat and cow milk and of colostrum of ewes and goats. Small Ruminant Research 18: 255-262. https: / / doi.org/10.1016/0921-4488(95)00697-3

Hayssen, V. 2009. Bradypus tridactylus (Pilosa: Bradypodidae). Mammalian Species 839: 1-9. https: / / doi.org / 10.1644/839.1

IDEAM. 2014. Boletín climatológico mensual. Instituto de Hidrología, Meteorología y Estudios Ambientales, Gobierno de Colombia <http: / / www.ideam.gov.co/ web / tiempo-yclima/climatologico-mensual> Consultada 1 de diciembre de 2016.

Mackenzie, A., G. Ernst \& Z. Taranu. 2005. Behavioural studies and rehabilitation of sloths in Parque Natural Metropolitano. Unpublished report, Smithsonian Tropical Research Institute, Panama. 67 pp.

Messias, A., A. K. C. Beresca, L. Munao \& C. Esbérard. 2001. Order Xenarthra (Edentata) (sloths, armadillos, anteaters). Pp. 238-255 in: Biology, medicine and surgery of South American wild animals (M. Fowler \& Z. Cubas, eds.). Iowa State University Press, Iowa.

Morales-Jiménez A., F. Sánchez, K. Poveda \& A. Cadena. 2004. Guía de mamíferos terrestres de Colombia. Bogotá, Colombia. 248 pp.

Mueller, R. \& F. Dethioux. 2010. Dermatosis nutricionales y la influencia de la nutrición en dermatología. Pp. 51-75 in: Enciclopedia de la nutrición clínica felina (P. Pibot, V. Biourge \& D. Elliott, eds.). Royal Canin, París.
Murata, K. \& M. Masuda. 1996. Gender determination of the Linne's two-toed sloth (Choloepus didactylus) using SRY amplified from hair. Journal of Veterinary Medical Science 58: 1157-1159. https:/ / doi.org/10.1292/jvms.58.12_1157

Plese, T. 2014. Los perezosos no son osos. Fundación AIUNAU, CVS - Corporación Autónoma Regional de los Valles del Sinú y San Jorge, Córdoba, Colombia. 55 pp.

Plese, T., S. Moreno \& C. Rodríguez. 2008. Estrategia Nacional para la prevención y control al tráfico ilegal de las especies silvestres de perezosos en Colombia. Ministerio de Ambiente, Vivienda y Desarrollo Territorial, Fundación Unau y Corantioquia, Bogotá. 24 pp.

Plese, T., N. Reyes-Amaya, L. Castro-Vásquez, S. Giraldo \& O. Feliciano. 2016. Distribution and current state of knowledge of Hoffmann's twotoed sloth (Choloepus hoffmanni) in Colombia, with comments on the variations of its external morphological traits. Therya 7: 407-422. https:/ / doi.org/10.12933/ therya-16-412

Taube, E., J. Keravec, J. C. Vié \& J. Duplantier. 2001. Reproductive biology and postnatal development in sloths, Bradypus and Choloepus: review with original data from the field (French Guiana) and from captivity. Mammal Review 31: 173-188. https: / / doi. org/10.1046/j.1365-2907.2001.00085.x

Varela, N. 2009. Normas editoriales para publicaciones de la Asociación de Veterinarios de Vida Silvestre. Asociación de Veterinarios de Vida Silvestre, Pereira, Colombia. 24 pp.

Recibido: 21 de septiembre de 2016; Aceptado: 12 de diciembre de 2016 